\title{
Combined effects of knee extension strength, visual acuity, and knee-joint pain on older women's gait
}

\author{
Tomohiro Demura $^{1^{*}}$, Shinichi Demura ${ }^{2}$ \\ ${ }^{1}$ Child Education, Jinai Women’s College, Fukui, Japan; *Corresponding Author: tomodemu@jin-ai.ac.jp \\ ${ }^{2}$ Graduate School of Natural Science \& Technology, Kanazawa University, Kanazawa, Japan
}

Received 22 January 2013; revised 24 February 2013; accepted 3 March 2013

\begin{abstract}
The purpose of this study was to examine the combined effects of knee extension strength (KES), visual acuity (VA), and knee joint pain (KP) on gait in 212 older women. Including, walking speed, cadence, were selected as gait parameters. Knee extension strength was measured by isometric knee extension strength, while knee joint pain and decreased visual acuity were evaluated by subjective judgment. The combine effect of KP and KES factors was examined. Stance time was significantly longer in persons with both-KP than in persons with no KP. In addition, people with superior KES had significantly greater values in walking speed, cadence, and step length, and lower values in stance time and walking angle than those with inferior KES. Furthermore, double support time showed that persons with both-KP have significantly greater values than persons with no or one-KP in the inferior KES group. Also, persons with the inferior KES had significantly greater values in persons with both-KP. The combine effect of KES and VA factors was examined. There are significant differences between the superior and the inferior KES groups. In conclusion, the elderly with both the factors of decreasing KES and both-KP, as compared to the elderly with just one of those factors, have markedly different gait properties.
\end{abstract}

Keywords: Community-Dwelling Elderly; Gait; Knee Joint Pain; Vision Acuity

\section{INTRODUCTION}

Recently, the elderly population in Japan has greatly increased, resulting in a greater interest in the health problems of the elderly. Often, when the elderly fall, it causes fracturing of the femoral neck [1], and thus requires them to enter a care facility [2], which, in turn, causes a decrease in healthy life expectancy, or QOL
(Quality of Life). Risk factors for falls include advanced age (aged 80 years or older), an abnormal gait, a decrease in balance ability, cognitive impairment, and/or a diagnosis of Parkinson's disease [3]. Falls occur mostly while walking; therefore, walking is one of the fundamental daily living activities that has been frequently studied.

The following changes in walking occur as a person ages: a decrease in walking speed $[4,5]$, a change in the ratio of single leg and double leg support times in the total contact time [6], and a decreased step length [6]. It was reported that these gait changes in old age are also related to falls [7-9]. In addition, a person's gait has been used as an indicator for predicting the risk of disease and death among the elderly [8].

Decreases in various physical functions such as leg strength, leg joint performance, and visual acuity affect gait in old age. Specifically, there are many reports on the effects of decreased leg strength on gait [3,10-12]. Additionally, visual information is important for stable walking, and a decrease in visual function (e.g., low visual acuity) inhibits the achievement of daily living activities [13]. According to Elliott et al. [14] and MoeNilssen et al. [15], temporary restriction of visual information induces gait change. It was reported that a decrease in visual acuity in the elderly increases the risk of falls $[2,16,17]$.

On the other hand, leg joints play an important role in gait because walking is a movement that mainly uses the lower limbs. Knee joint function decreases in old age, and patients with knee osteoarthritis have slower walking speed and a slower cadence, or shorter step length, and longer stance time[18,19]. Kaufman et al. [20] confirmed that knee osteoarthritis patients, as compared with healthy subjects, walk with a shorter knee extension moment to reduce knee strain. Generally, the elderly have decreased joint function. About $25 \%$ of the elderly aged 55 or over, including knee osteoarthritis patients, complain of knee joint pain $[21,22]$. Knee joint pain greatly affects walking [23]. Noro et al. [24] pointed out that mild knee pain may lead to "gait disorder", which causes an inability to walk smoothly.

Thus vision problems and knee joint pain, in addition 
to decreased leg strength, are considered to be important factors affecting gait. In addition, it is assumed that persons with multiple factors at once experience more difficulty walking than persons with one factor or none.

This study aimed to examine the combined effects of knee extension strength, visual acuity, and knee joint pain on gait in elderly women.

\section{MATERIALS AND METHODS}

\subsection{Subjects}

The subjects were 212 healthy, community-dwelling older women. Table 1 shows their age and physical characteristics. All subjects participated in health classes and/ or social educational activities that were hosted by municipal governments. They were judged to have high independence in daily activities because many of them engaged in leisure activity and work. Before the measurements were made, the purpose and procedure of this study were explained in detail and informed consent was obtained from all subjects. In addition, this study was approved by the Ethics Committee on Human Experimentation of Faculty of Education, Kanazawa University (Ref. No. 19-17).

\subsection{Testing Protocol}

\subsubsection{Gait Property}

Gait properties were measured by the gait analysis system Walk Way MG-1000 (Anima, Japan) referenced in Demura \& Demura's [25] method. The MG-1000 with plate sensors can determine the time, dimensions and distance of the foot or feet when touching its surface, and measure grounding/non-grounding on the bearing surface as an on/off signal. Data was recorded into a personal computer at $100 \mathrm{~Hz}$. Subjects walked a 12 meter path with the above sensors at voluntary maximum speed. The analysis interval was approximately $4 \mathrm{~m}$ to $8 \mathrm{~m}$.

\subsubsection{Factors}

\section{1) Leg Strength}

Table 1. Characteristics of subjects (Female: 212).

\begin{tabular}{cccccc}
\hline $\mathrm{n}=212$ & & Mean & SD & Max & Min \\
\hline Age & (years) & 76.1 & 5.75 & 93.0 & 62.0 \\
Height & $(\mathrm{cm})$ & 147.1 & 5.72 & 161.4 & 129.0 \\
Weight & $(\mathrm{kg})$ & 49.8 & 7.68 & 70.9 & 31.0 \\
$\begin{array}{c}\text { Knee extension } \\
\text { strength }\end{array}$ & $(\mathrm{kg})$ & 6.8 & 2.05 & 13.5 & 1.9 \\
$\begin{array}{c}\text { Prevalence of visual } \\
\text { acuity problems }\end{array}$ & $(\%)$ & With: 48 (23\%) & $\begin{array}{c}\text { Without: } 164 \\
(77 \%)\end{array}$ \\
$\begin{array}{c}\text { Prevalence of knee } \\
\text { pain }\end{array}$ & $(\%)$ & One: 71 (34\%), Both: 45 (21\%), No: \\
\hline
\end{tabular}

With/Without: Persons with/without visual acuity problem, One/Both/No: Persons with one-/both-/no knee pain.
Knee extension strength was selected for assessing leg strength [26]. During measurement of isometric knee extension strength, the subjects were seated upright in a rigid chair with knees flexed at an angle of $90^{\circ}$, and with the lower legs strapped in a pad just above the ankle and held in place by a backward rigid bar to a tension meter attachment (T.K.K.1269f; Takei Scientific Instruments Co. Ltd., Japan). In addition, the subjects folded their arms on their chest. A tester adjusted the pad so as not to move, and the subject was asked to extend the knee as far as possible and to maintain it for 3 seconds. Each of the subjects' legs was measured twice at intervals greater than thirty seconds. A mean of their values was used as an evaluation value. Based on mean value (Mean), subjects were divided into two groups with different knee extension strength (Inferior group: Inferior score < Mean, Superior group: Superior score $>$ Mean).

2) Visual Acuity

In old age, a decrease in visual function greatly affects gait. Persons with visual acuity problems selected in this study had difficulty reading characters or seeing people's faces, and also felt hindered in their gait (with visual acuity problem group). The visual acuity problems were examined in advance.

These indicators of visual acuity were also used in a fall risk survey [27].

3) Knee Joint Pain

Knee joints with orthopedic disorders affect walking $[18,19]$. It was also assumed that subjective knee pain affects walking [23]. Knee problems were examined before measurement. Subjects were divided into those with pain in one knee joint (unilateral knee pain group) those with pain in both knee joints (bilateral knee pain group), and those without knee pain (non-knee pain group).

\subsection{Parameters}

\section{Gait Variability (Temporal and Spatial Parameters)}

The following 9 gait parameters were selected in reference to previous studies [25]: walking speed, cadence, stance time, swing time, double support time, step length, step width, walking angle, and toe angle.

Walking speed: walking distance per second ( $\mathrm{cm} / \mathrm{sec})$.

Cadence: the number of steps per minute (steps/min).

Stance time: the duration that the body is supported by one or both feet, that is, the phase in which one foot or both feet contact the floor.

Swing time: the duration that one foot swings, that is, one foot leaves the floor. This time agrees with a single support time (sec).

Double support time: the duration in which both feet contact the floor (sec).

Step length: the distance between anterior-posterior patterns (one step length). 
Step width: the distance between both feet $(\mathrm{cm})$.

Walking angle: the angle between the direction of movement and the bilateral pattern line $\left({ }^{\circ}\right)$.

Toe angle: the angle between the direction of movement and the foot axis $\left({ }^{\circ}\right)$.

\subsection{Data Analysis}

The elderly generally have a decrease in knee extension strength and vision problems or knee joint pain simultaneously. In this study, focusing on the above three factors, the effect of two combined factors on gait was examined when controlling the third factor.

\subsubsection{The Combined Effect of Visual Acuity and Knee Pain on Gait}

When controlling the effect of knee extension strength (analyze as covariate), the differences in mean values among six groups according to visual acuity factor (with and without visual acuity problem) and knee joint pain factor (unilateral, bilateral, and non-knee pain) for gait parameters were examined by unpaired two-way analysis of variance.

\subsubsection{The Combined Effect of Knee Extension Strength and Knee Pain on Gait}

For the elderly without a visual acuity problem, the differences in mean values among six groups according to knee joint pain factor (unilateral, bilateral, and nonknee pain), and knee extension strength factor (the superior and inferior knee extension strength) for gait parameters were examined by unpaired two-way analysis of variance.

\subsubsection{The Combined Effect of Knee Extension Strength and Visual Acuity on Gait}

For the elderly with non-knee pain, the differences in mean values among four groups according to visual acuity factor (with and without visual acuity problem), and knee extension strength factor (the superior and inferior knee extension strength) for gait parameters were examined by unpaired two-way analysis of variance.

In addition, if a significant difference was found in age, height, and weight, gait parameters were tested using analysis of covariance. The Bonferroni method was selected for multiple comparisons. The statistical SPSS package ver. 11.0 (SPSS, America) was used for data analysis. A probability level of 0.05 was indicative of statistical significance.

\section{RESULTS}

\subsection{The Combined Effect of Visual Acuity and Knee Pain on Gait}

Table 2 shows the basic statistics and results of un- paired two-way analyses of variance for gait parameters of six groups according to visual acuity factor (with and without visual acuity problem), knee joint pain factor (unilateral, bilateral , and non-knee pain), and controlled effect of knee extension strength (analyze as covariate). Because a significant difference was found in body weight, analysis of covariance was used. There was no significant difference among groups.

\subsection{The Combined Effect of Knee Extension Strength and Knee Pain on Gait}

Table 3 shows the basic statistics of six groups according to knee joint pain factor (unilateral, bilateral, and non-knee pain) and knee extension strength factor (superior and inferior knee extension strength), and the results of unpaired two-way analysis of variance for gait parameters in the elderly without visual acuity problems. Since significant differences were found in age and body weight, analysis of covariance was used. Stance time and double support time showed significant primary effect in $\mathrm{KP}$, and multiple comparisons showed that stance time is longer in persons with bilateral pain as opposed to persons with no pain. In addition, significant primary effects were found in walking speed, cadence, stance time, double support time, step length, and walking angle in the knee extension strength factor. The superior KES subjects scored higher values in walking speed, cadence, and step length and lower values in stance time and walking angle, as compared with the inferior KES group.

Double support time showed significant interaction, and multiple comparisons revealed that in the inferior KES group, it was longer in subjects with bilateral KP than those with unilateral KP or non-pain, and that it was longer in the subjects with bilateral KP in the inferior KES group than those in the superior KES group with bilateral KP.

\subsection{The Combined Effect of Knee Extension Strength and Visual Acuity on Gait}

Table 4 shows the basic statistics of four groups according to visual acuity factor (with and without visual acuity problems) and knee extension strength factor (the superior and inferior knee extension strength), and the results of unpaired two-way analysis of variance for gait parameters in the non-knee pain group. Since a significant difference was found in body weight, analysis of covariance was used. Except for toe angle, all parameters showed significant differences in the KES factor, and compared to the inferior KES group, the superior KES group's results show larger values for walking speed, cadence, and step length, and smaller values for stance time, swing time, double support time, step width, and walking angle. 
Table 2. The basic statistics and results of two-way analysis of variance for gait parameters among six groups according to visual acuity and knee joint pain factors, considered effect of knee extension strength.

\begin{tabular}{|c|c|c|c|c|c|c|c|c|c|c|c|c|c|}
\hline \multirow{2}{*}{\multicolumn{2}{|c|}{$\begin{array}{c}\text { Visual acuity problem } \\
\text { Knee pain }\end{array}$}} & \multirow{2}{*}{$\frac{1}{1}$} & \multicolumn{3}{|c|}{ With } & \multicolumn{3}{|c|}{ Without } & \multicolumn{3}{|c|}{2 way-ANOVA } & \multicolumn{2}{|c|}{ ANCOVA } \\
\hline & & & One & Both & No & One & Both & No & & F value & $\mathrm{p}$ value & F value & $\mathrm{p}$ value \\
\hline \multirow{4}{*}{$\begin{array}{l}\text { Knee extension } \\
\text { strength }\end{array}$} & \multirow{4}{*}{ (kg) } & \multirow{4}{*}{$\begin{array}{l}\text { Mean } \\
\text { SD }\end{array}$} & $\mathrm{n}=17$ & $\mathrm{n}=8$ & $n=23$ & $\mathrm{n}=54$ & $\mathrm{n}=37$ & \multicolumn{2}{|l|}{$\mathrm{n}=73$} & & & \multirow{4}{*}{\multicolumn{2}{|c|}{ covariate }} \\
\hline & & & 5.79 & 7.33 & 7.14 & 6.74 & 6.14 & 7.16 & $\mathrm{~F} 1$ & 0.04 & 0.84 & & \\
\hline & & & 1.65 & 1.73 & 1.96 & 2.11 & 2.10 & 2.04 & $\mathrm{~F} 2$ & 2.84 & 0.06 & & \\
\hline & & & & & & & & & F3 & 2.48 & 0.09 & & \\
\hline \multirow{3}{*}{ Age } & \multirow{3}{*}{ (years) } & \multirow{3}{*}{$\begin{array}{l}\text { Mean } \\
\text { SD }\end{array}$} & \multirow{3}{*}{$\begin{array}{c}75.5 \\
5.0\end{array}$} & \multirow{3}{*}{$\begin{array}{c}74.6 \\
1.8\end{array}$} & 76.1 & 75.7 & 77.4 & 76.1 & $\mathrm{~F} 1$ & 0.90 & 0.34 & & \\
\hline & & & & & 5.8 & 5.1 & 5.5 & 6.7 & $\mathrm{~F} 2$ & 0.58 & 0.56 & & \\
\hline & & & & & & & & & F3 & 0.29 & 0.75 & & \\
\hline & & Mean & 146.0 & 146.6 & 147.1 & 148.1 & 147.8 & 146.4 & $\mathrm{~F} 1$ & 0.83 & 0.36 & & \\
\hline Height & $(\mathrm{cm})$ & SD & 4.9 & 4.7 & 6.4 & 5.7 & 6.0 & 5.7 & $\mathrm{~F} 2$ & 0.18 & 0.84 & & \\
\hline & & & & & & & & & F3 & 0.83 & 0.44 & & \\
\hline & & Mean & 50.8 & 52.5 & 47.7 & 51.9 & 52.0 & 47.4 & $\mathrm{~F} 1$ & 0.02 & 0.89 & & \\
\hline Weight & (kg) & SD & 8.4 & 5.0 & 8.7 & 7.9 & 7.0 & 7.0 & $\mathrm{~F} 2$ & 8.92 & $0.00^{*}$ & $\operatorname{cov}$ & riate \\
\hline & & & & & & & & & F3 & 0.09 & 0.92 & & \\
\hline & & Mean & 150.4 & 168.0 & 162.8 & 165.8 & 152.9 & 170.0 & $\mathrm{~F} 1$ & - & - & 0.35 & 0.56 \\
\hline $\begin{array}{c}\text { Walking } \\
\text { speed }\end{array}$ & $(\mathrm{cm} / \mathrm{sec})$ & $\mathrm{SD}$ & 29.0 & 26.8 & 32.7 & 28.7 & 35.5 & 32.8 & $\mathrm{~F} 2$ & - & - & 0.14 & 0.87 \\
\hline & & & & & & & & & F3 & - & - & 0.70 & 0.50 \\
\hline & & Mean & 153.2 & 160.2 & 156.9 & 162.2 & 153.5 & 161.6 & $\mathrm{~F} 1$ & - & - & 0.51 & 0.48 \\
\hline Cadence & (steps/min) & SD & 18.2 & 15.3 & 19.6 & 24.1 & 21.7 & 19.6 & $\mathrm{~F} 2$ & - & - & 0.12 & 0.88 \\
\hline & & & & & & & & & F3 & - & - & 0.47 & 0.62 \\
\hline & & Mean & 0.47 & 0.44 & 0.45 & 0.43 & 0.47 & 0.43 & $\mathrm{~F} 1$ & - & - & 0.81 & 0.37 \\
\hline Stance time & (sec) & SD & 0.07 & 0.05 & 0.07 & 0.07 & 0.08 & 0.06 & F2 & - & - & 0.24 & 0.79 \\
\hline & & & & & & & & & F3 & - & - & 0.99 & 0.37 \\
\hline & & Mean & 0.33 & 0.32 & 0.34 & 0.33 & 0.33 & 0.33 & $\mathrm{~F} 1$ & - & - & 0.06 & 0.80 \\
\hline Swing time & (sec) & SD & 0.03 & 0.03 & 0.03 & 0.04 & 0.04 & 0.03 & $\mathrm{~F} 2$ & - & - & 0.32 & 0.72 \\
\hline & & & & & & & & & F3 & - & - & 0.46 & 0.63 \\
\hline Double & & Mean & 0.07 & 0.06 & 0.06 & 0.06 & 0.07 & 0.05 & $\mathrm{~F} 1$ & - & - & 1.74 & 0.19 \\
\hline support & (sec) & SD & 0.02 & 0.02 & 0.03 & 0.02 & 0.03 & 0.02 & F2 & - & - & 0.50 & 0.61 \\
\hline & & & & & & & & & F3 & - & - & 0.97 & 0.38 \\
\hline & & Mean & 58.6 & 62.8 & 61.9 & 61.5 & 59.4 & 63.0 & $\mathrm{~F} 1$ & - & - & 0.05 & 0.82 \\
\hline Step length & $(\mathrm{cm})$ & SD & 8.0 & 6.7 & 8.3 & 7.8 & 9.3 & 9.1 & $\mathrm{~F} 2$ & - & - & 0.28 & 0.75 \\
\hline & & & & & & & & & F3 & - & - & 0.36 & 0.70 \\
\hline & & Mean & 7.66 & 7.10 & 7.79 & 7.27 & 8.19 & 7.15 & $\mathrm{~F} 1$ & - & - & 0.00 & 0.99 \\
\hline Step width & $(\mathrm{cm})$ & SD & 2.58 & 2.08 & 2.69 & 2.48 & 2.58 & 2.51 & $\mathrm{~F} 2$ & - & - & 0.35 & 0.70 \\
\hline & & & & & & & & & F3 & - & - & 0.76 & 0.47 \\
\hline & & Mean & 7.87 & 6.56 & 7.36 & 7.00 & 8.20 & 6.67 & $\mathrm{~F} 1$ & - & - & 0.00 & 0.98 \\
\hline $\begin{array}{l}\text { Walking } \\
\text { angle }\end{array}$ & $\left({ }^{\circ}\right)$ & SD & 3.35 & 2.41 & 2.97 & 2.71 & 3.31 & 2.74 & $\mathrm{~F} 2$ & - & - & 0.03 & 0.97 \\
\hline & & & & & & & & & F3 & - & - & 1.03 & 0.36 \\
\hline & & Mean & 4.52 & 2.09 & 2.70 & 3.01 & 2.96 & 2.91 & $\mathrm{~F} 1$ & - & - & 0.04 & 0.85 \\
\hline Toe angle & $\left({ }^{\circ}\right)$ & SD & 3.39 & 1.97 & 4.10 & 4.02 & 4.08 & 3.55 & F2 & - & - & 1.66 & 0.19 \\
\hline & & & & & & & & & F3 & - & - & 1.40 & 0.25 \\
\hline
\end{tabular}

With/Without: Persons with/without visual acuity problem, One/Both/No: Persons with one-/both-/no knee pain, F1: Visual acuity problem, F2: Knee pain, F3: Interaction, ${ }^{*}: \mathrm{p}<0.05$. 
Table 3. The basic statistics and results of two-way analysis of variance for gait parameters among six groups according to knee joint pain and knee extension strength factor for "without acuity problem group”.

\begin{tabular}{|c|c|c|c|c|c|c|c|c|c|c|c|c|c|c|}
\hline \multirow{2}{*}{\multicolumn{2}{|c|}{$\begin{array}{l}\text { Knee pain } \\
\text { Knee extension strength }\end{array}$}} & \multirow{2}{*}{ / } & \multicolumn{2}{|c|}{ One } & \multicolumn{2}{|c|}{ Both } & \multicolumn{3}{|c|}{ No } & \multicolumn{2}{|c|}{ ANOVA } & \multicolumn{2}{|c|}{ ANCOVA } & \multirow{2}{*}{$\begin{array}{l}\text { Post-hoc } \\
\text { Bonferoni }\end{array}$} \\
\hline & & & $\begin{array}{l}\text { Sup } \\
\mathrm{n}=23\end{array}$ & $\begin{array}{c}\text { Inf } \\
\mathrm{n}=31\end{array}$ & $\begin{array}{c}\text { Sup } \\
\mathrm{n}=17\end{array}$ & $\begin{array}{c}\text { Inf } \\
\mathrm{n}=20\end{array}$ & $\begin{array}{c}\text { Sup } \\
\mathrm{n}=36\end{array}$ & $\begin{array}{c}\text { Inf } \\
\mathrm{n}=37\end{array}$ & & F value & $\mathrm{p}$ value & F value & $\mathrm{p}$ value & \\
\hline \multirow{3}{*}{ Age } & \multirow{3}{*}{ (years) } & Mean & 74.4 & 76.7 & 74.7 & 79.7 & 75.6 & 76.6 & $\mathrm{~F} 1$ & 0.84 & 0.43 & & & \multirow{6}{*}{ covariate } \\
\hline & & SD & 4.37 & 5.50 & 5.14 & 4.86 & 6.75 & 6.77 & $\mathrm{~F} 2$ & 8.32 & $0.00^{*}$ & & & \\
\hline & & & & & & & & & F3 & 1.35 & 0.26 & & & \\
\hline \multirow{3}{*}{ Height } & \multirow{3}{*}{$(\mathrm{cm})$} & Mean & 148.3 & 148.0 & 148.5 & 147.2 & 146.4 & 146.4 & $\mathrm{~F} 1$ & 1.55 & 0.22 & - & - & \\
\hline & & \multirow{2}{*}{ SD } & \multirow[t]{2}{*}{4.99} & \multirow[t]{2}{*}{6.26} & \multirow[t]{2}{*}{5.32} & \multirow[t]{2}{*}{6.58} & \multirow[t]{2}{*}{6.89} & \multirow[t]{2}{*}{4.31} & $\mathrm{~F} 2$ & 0.32 & 0.58 & - & - & \\
\hline & & & & & & & & & F3 & 0.16 & 0.86 & - & - & \\
\hline \multirow{3}{*}{ Weight } & & Mean & 53.0 & 51.0 & 55.6 & 49.0 & 48.6 & 46.3 & $\mathrm{~F} 1$ & 8.87 & $0.00^{*}$ & & & \\
\hline & (kg) & SD & 6.46 & 8.77 & 7.21 & 5.17 & 7.73 & 6.05 & $\mathrm{~F} 2$ & 9.94 & $0.00^{*}$ & & & covariate \\
\hline & & & & & & & & & F3 & 1.36 & 0.26 & & & \\
\hline Knee & & Mean & 8.68 & 5.31 & 8.05 & 4.52 & 8.72 & 5.65 & $\mathrm{~F} 1$ & - & - & 8.59 & $0.00^{*}$ & One, No > Both \\
\hline extension & (kg) & $\mathrm{SD}$ & 1.31 & 1.27 & 1.22 & 1.02 & 1.55 & 1.11 & $\mathrm{~F} 2$ & - & - & 217.51 & $0.00^{*}$ & Sup $>$ Inf \\
\hline & & & & & & & & & F3 & - & - & 0.24 & 0.79 & сар - 1111 \\
\hline & & Mean & 174.5 & 159.4 & 175.4 & 133.8 & 181.6 & 158.8 & $\mathrm{~F} 1$ & - & - & 2.43 & 0.09 & \\
\hline $\begin{array}{l}\text { Walking } \\
\text { speed }\end{array}$ & $(\mathrm{cm} / \mathrm{sec})$ & SD & 31.7 & 24.8 & 28.0 & 29.9 & 29.5 & 32.3 & $\mathrm{~F} 2$ & - & - & 21.41 & $0.00^{*}$ & Sup $>$ Inf \\
\hline & & & & & & & & & F3 & - & - & 1.71 & 0.18 & \\
\hline & & Mean & 166.4 & 159.1 & 163.9 & 144.7 & 167.1 & 156.2 & $\mathrm{~F} 1$ & - & - & 1.52 & 0.22 & \\
\hline Cadence & (steps/min) & SD & 22.7 & 25.0 & 21.1 & 18.4 & 19.1 & 18.8 & F2 & - & - & 9.61 & $0.00^{*}$ & Sup $>$ Inf \\
\hline & & & & & & & & & F3 & - & - & 0.69 & 0.51 & \\
\hline & & Mean & 0.42 & 0.44 & 0.43 & 0.51 & 0.41 & 0.45 & $\mathrm{~F} 1$ & - & - & 3.69 & $0.03^{*}$ & Both $>$ No \\
\hline Stance time & (sec) & SD & 0.07 & 0.06 & 0.06 & 0.08 & 0.06 & 0.07 & F2 & - & - & 14.50 & $0.00^{*}$ & Sun \\
\hline & & & & & & & & & F3 & - & - & 1.99 & 0.14 & सात \\
\hline & & Mean & 0.32 & 0.33 & 0.33 & 0.34 & 0.32 & 0.34 & $\mathrm{~F} 1$ & - & - & 0.36 & 0.70 & \\
\hline Swing time & (sec) & SD & 0.04 & 0.04 & 0.03 & 0.05 & 0.03 & 0.03 & $\mathrm{~F} 2$ & - & - & 3.73 & 0.06 & \\
\hline & & & & & & & & & F3 & - & - & 0.19 & 0.83 & \\
\hline Double & & Mean & 0.05 & 0.06 & 0.05 & 0.08 & 0.05 & 0.06 & $\mathrm{~F} 1$ & - & - & 4.02 & $0.02^{*}$ & $\begin{array}{c}\text { Inf: Both }>\text { One, } \\
\text { No }\end{array}$ \\
\hline $\begin{array}{l}\text { support } \\
\text { time }\end{array}$ & (sec) & SD & 0.02 & 0.02 & 0.02 & 0.04 & 0.02 & 0.02 & F2 & - & - & 17.70 & $0.00^{*}$ & Both: Inf > Sup \\
\hline & & & & & & & & & F3 & - & - & 4.14 & $0.02^{*}$ & \\
\hline & & Mean & 62.9 & 60.5 & 64.2 & 55.3 & 65.3 & 60.9 & $\mathrm{~F} 1$ & - & - & 1.29 & 0.28 & \\
\hline Step length & $(\mathrm{cm})$ & SD & 7.76 & 7.72 & 5.85 & 9.76 & 7.91 & 9.66 & F2 & - & - & 8.81 & $0.00^{*}$ & Sup $>$ Inf \\
\hline & & & & & & & & & F3 & - & - & 1.25 & 0.29 & \\
\hline & & Mean & 6.81 & 7.61 & 7.91 & 8.42 & 6.95 & 7.34 & $\mathrm{~F} 1$ & - & - & 1.74 & 0.18 & \\
\hline Step width & $(\mathrm{cm})$ & SD & 2.83 & 2.17 & 2.58 & 2.62 & 2.21 & 2.79 & F2 & - & - & 1.55 & 0.21 & \\
\hline & & & & & & & & & F3 & - & - & 0.08 & 0.92 & \\
\hline & & Mean & 6.48 & 7.39 & 7.12 & 9.12 & 6.25 & 7.07 & $\mathrm{~F} 1$ & - & - & 2.41 & 0.09 & \\
\hline $\begin{array}{l}\text { Walking } \\
\text { angle }\end{array}$ & $\left({ }^{\circ}\right)$ & SD & 3.12 & 2.34 & 2.34 & 3.76 & 2.37 & 3.04 & $\mathrm{~F} 2$ & - & - & 5.59 & $0.02^{*}$ & Inf $>$ Sup \\
\hline & & & & & & & & & F3 & - & - & 0.45 & 0.64 & \\
\hline & & Mean & 2.99 & 3.02 & 2.77 & 3.12 & 2.91 & 2.92 & $\mathrm{~F} 1$ & - & - & 0.01 & 0.99 & \\
\hline Toe angle & $\left({ }^{\circ}\right)$ & SD & 4.05 & 4.07 & 3.49 & 4.60 & 3.24 & 3.88 & F2 & - & - & 0.16 & 0.69 & \\
\hline & & & & & & & & & F3 & - & - & 0.05 & 0.95 & \\
\hline
\end{tabular}

Sup/Inf: Persons with the superior/inferior knee extension strength, One/Both/No: Persons with one-/both-/no knee pain, F1: Knee pain, F2: Knee extension strength, F3: Interaction, ${ }^{*}: \mathrm{p}<0.05$. 
Table 4. The basic statistics and results of two-way analysis of variance for gait parameters among four groups according to visual acuity and knee extension strength factors for "non-knee pain group".

\begin{tabular}{|c|c|c|c|c|c|c|c|c|c|c|c|c|}
\hline \multirow{2}{*}{\multicolumn{2}{|c|}{$\begin{array}{c}\text { Visual acuity problem } \\
\text { Knee extension strength }\end{array}$}} & \multicolumn{2}{|c|}{ I } & \multirow{2}{*}{$\begin{array}{c}\text { With } \\
\mathrm{Inf} \\
\mathrm{n}=12\end{array}$} & \multicolumn{2}{|c|}{ Without } & & \multicolumn{2}{|c|}{ ANOVA } & \multicolumn{2}{|c|}{ ANCOVA } & \multirow{2}{*}{$\begin{array}{l}\text { Post-hoc } \\
\text { Bonferon }\end{array}$} \\
\hline & & / & $\begin{array}{c}\text { Sup } \\
\mathrm{n}=11\end{array}$ & & $\begin{array}{c}\text { Sup } \\
\mathrm{n}=36\end{array}$ & $\begin{array}{c}\text { Inf } \\
\mathrm{n}=37\end{array}$ & & $\mathrm{~F}$ & $\mathrm{p}$ & $\mathrm{F}$ value & $\mathrm{p}$ value & \\
\hline \multirow{3}{*}{ Age } & & Mean & 74.2 & 77.8 & 75.6 & 76.6 & $\mathrm{~F} 1$ & 0.00 & 0.97 & - & - & \\
\hline & (years) & SD & 6.59 & 4.49 & 6.75 & 6.77 & F2 & 2.27 & 0.14 & - & - & \\
\hline & & & & & & & F3 & 0.70 & 0.40 & - & - & \\
\hline \multirow{3}{*}{ Height } & & Mean & 147.5 & 146.7 & 146.4 & 146.4 & $\mathrm{~F} 1$ & 0.22 & 0.64 & - & - & \\
\hline & $(\mathrm{cm})$ & SD & 6.58 & 6.55 & 6.89 & 4.31 & F2 & 0.08 & 0.78 & - & - & \\
\hline & & & & & & & F3 & 0.07 & 0.79 & - & - & \\
\hline \multirow{3}{*}{ Weight } & & Mean & 50.5 & 45.2 & 48.6 & 46.3 & F1 & 0.06 & 0.80 & & & \\
\hline & (kg) & SD & 8.23 & 8.58 & 7.73 & 6.05 & F2 & 4.81 & $0.03^{*}$ & & & covariate \\
\hline & & & & & & & F3 & 0.75 & 0.39 & & & \\
\hline \multirow{3}{*}{$\begin{array}{l}\text { Knee extension } \\
\text { strength }\end{array}$} & & Mean & 8.59 & 5.82 & 8.72 & 5.65 & F1 & - & - & 0.00 & 1.00 & \\
\hline & (kg) & SD & 1.49 & 1.29 & 1.55 & 1.11 & $\mathrm{~F} 2$ & - & - & 71.89 & $0.00^{*}$ & Sup > Inf \\
\hline & & & & & & & F3 & - & - & 0.42 & 0.52 & \\
\hline \multirow{3}{*}{ Walking speed } & & Mean & 180.0 & 147.1 & 181.6 & 158.8 & F1 & - & - & 0.89 & 0.35 & \\
\hline & $(\mathrm{cm} / \mathrm{sec})$ & SD & 24.6 & 31.9 & 29.5 & 32.3 & $\mathrm{~F} 2$ & - & - & 12.01 & $0.00^{*}$ & Sup > Inf \\
\hline & & & & & & & F3 & - & - & 0.33 & 0.57 & \\
\hline \multirow{3}{*}{ Cadence } & & Mean & 165.4 & 149.0 & 167.1 & 156.2 & F1 & - & - & 1.00 & 0.32 & \\
\hline & (steps/min) & SD & 16.1 & 19.8 & 19.1 & 18.8 & F2 & - & - & 8.16 & $0.01^{*}$ & Sup $>$ Inf \\
\hline & & & & & & & F3 & - & - & 0.32 & 0.57 & \\
\hline \multirow{3}{*}{ Stance time } & & Mean & 0.41 & 0.48 & 0.41 & 0.45 & F1 & - & - & 1.60 & 0.21 & \\
\hline & (sec) & SD & 0.05 & 0.08 & 0.06 & 0.07 & $\mathrm{~F} 2$ & - & - & 10.32 & $0.00^{*}$ & Inf $>$ Sup \\
\hline & & & & & & & F3 & - & - & 1.18 & 0.28 & \\
\hline \multirow{3}{*}{ Swing time } & & Mean & 0.33 & 0.34 & 0.32 & 0.34 & F1 & - & - & 0.24 & 0.63 & \\
\hline & (sec) & SD & 0.03 & 0.03 & 0.03 & 0.03 & F2 & - & - & 4.10 & $0.05^{*}$ & Inf $>$ Sup \\
\hline & & & & & & & F3 & - & - & 0.00 & 0.97 & \\
\hline \multirow{3}{*}{$\begin{array}{l}\text { Double support } \\
\text { time }\end{array}$} & & Mean & 0.05 & 0.07 & 0.05 & 0.06 & F1 & - & - & 1.62 & 0.21 & \\
\hline & (sec) & SD & 0.02 & 0.03 & 0.02 & 0.02 & F2 & - & - & 10.41 & $0.00^{*}$ & Inf $>$ Sup \\
\hline & & & & & & & F3 & - & - & 2.53 & 0.12 & \\
\hline \multirow{3}{*}{ Step length } & & Mean & 65.4 & 58.8 & 65.3 & 60.9 & $\mathrm{~F} 1$ & - & - & 0.25 & 0.62 & \\
\hline & $(\mathrm{cm})$ & SD & 7.54 & 7.99 & 7.91 & 9.66 & F2 & - & - & 5.48 & 0.02 & Sup $>$ Inf \\
\hline & & & & & & & F3 & - & - & 0.18 & 0.67 & \\
\hline \multirow{3}{*}{ Step width } & & Mean & 6.70 & 8.79 & 6.95 & 7.34 & F1 & - & - & 0.95 & 0.33 & \\
\hline & $(\mathrm{cm})$ & SD & 2.49 & 2.56 & 2.21 & 2.79 & $\mathrm{~F} 2$ & - & - & 4.55 & 0.04 & Inf $>$ Sup \\
\hline & & & & & & & F3 & - & - & 2.09 & 0.15 & \\
\hline \multirow{3}{*}{ Walking angle } & & Mean & 5.90 & 8.71 & 6.25 & 7.07 & F1 & - & - & 0.96 & 0.33 & \\
\hline & $\left(^{\circ}\right)$ & SD & 2.49 & 2.80 & 2.37 & 3.04 & F2 & - & - & 7.70 & $0.01^{*}$ & Inf $>$ Sup \\
\hline & & & & & & & F3 & - & - & 2.39 & 0.13 & \\
\hline \multirow{3}{*}{ Toe angle } & & Mean & 2.20 & 3.16 & 2.91 & 2.92 & F1 & - & - & 0.05 & 0.82 & \\
\hline & $\left({ }^{\circ}\right)$ & SD & 3.40 & 4.76 & 3.24 & 3.88 & $\mathrm{~F} 2$ & - & - & 0.08 & 0.78 & \\
\hline & & & & & & & F3 & - & - & 0.18 & 0.67 & \\
\hline
\end{tabular}

With/Without: Persons with/without visual acuity problem, Sup/Inf: Persons with the superior/inferior knee extension strength, F1: Visual acuity problem, F2: Knee extension strength, F3: Interaction, ${ }^{*}: \mathrm{p}<0.05$. 


\section{DISCUSSION}

There were significant differences in body weight among the groups in this study. For example, in the group without vision acuity problems, the superior knee extension subjects were heavier than the inferior persons, and in the three KP groups, the bilateral KP group was heaviest and the non-KP weighed the least, with the unilateral KP group's weight falling in the middle. Blain et al. [28] reported that in women 60 years or older, there is a significant relationship between knee extension strength and body weight. In addition, it was reported that an increase in weight affects osteoarthiris [29], and that being overweight imposes a large burden on knee joints [30]. Restriction of activities by knee pain may promote an increase in weight.

\subsection{The Combined Effect of Visual Acuity and Knee Pain on Gait}

The body weight of persons with unilateral or bilateral KP was heavier than that of persons with no knee pain. Also, in controlling the effect of weight, changes in visual acuity and knee pain factors were not found.

Walking speed decreased when young persons with simulated cataracts walked in dim lighting [14]. Furthermore, Moe-Nilssen et al. [15] reported that a sudden change from normal to marginal lighting induces gait change. In addition, it was confirmed that by wearing goggles that restrict vision, walking speed, cadence, and step length decrease, while stance time and step width increase (Demura, \& Demura, in print). On the other hand, it was reported that gait characteristics are nearly normalized due to the adaptation to darkness during the first 90 seconds, suggesting that the darkness itself does not cause the gait changes [15]. From the above, a gait change may occur by restricted visual information.

Therefore, temporary restriction of visual information may also affect gait. The results presented above showed that, in elderly subjects who felt that their walking is hampered by vision problems, lower VA may not, in fact, be a special factor that produces a marked gait change. However, in this study, the measurements were conducted on a flat walkway and there was no task involving an obstacle that needed to be visually recognized, so that design could explain how a low VA did not markedly affect gait.

Al-Zahrani, \& Bakheit [18] and Bejek et al. [19] reported that osteoarthiris patients show a decrease in walking speed and step length, and an increase in stance time. Moreover, Bejek et al. [19] reported that osteoarthritis patients show a more prominent gait change due to the effects of pain when walking at a faster speed as compared to a slower speed. It was assumed that knee pain affects gait, even without osteoarthiris. In this study, however, gait did not change despite walking at maximum speed, in both subjects with and without any knee pain. The present subjects were healthy elderly who can perform daily living activities independently. In addition, the walking distance used in this study was short (about $12 \mathrm{~m}$ ). Thus, these results may suggest the following: if the elderly can perform daily life activities independently, they can walk a short distance even with subjective KP.

From the above, a combined effect of visual acuity and knee joint pain factors on gait was not found in this experimental condition.

\subsection{The Combined Effect of Knee Extension Strength and Knee Pain on Gait}

In the inferior knee extension strength group, subjects with bilateral KP showed longer double support times than subjects with unilateral or no KP. Additionally, in the bilateral KP group, the inferior KES subjects showed longer double support times than the superior group. An increase in double support time was reported as one of the gait changes that coincides with aging [30,31], and it has been considered that these gait properties in the elderly—which differ from those of younger people-may contribute to their increased walking stability [32]. It was clarified that the elderly with bilateral knee joint pain as well as inferior knee extension strength have inferior gait ability and markedly show these gait properties.

In overall trends, the superior KES group, compared to the inferior group, had faster walking speed, more cadence, shorter stance time, wider step length, and a smalller walking angle. Previous studies [31,33] reported that gait properties of the elderly are characterized by a decrease in step length and an increase in double support time and step width. A decrease in leg strength is closely related to a decrease in walking speed, and it is considered to be a main cause of gait change with age $[34,35]$. Therefore, the present results may also support the findings of previous studies.

\subsection{The Combined Effect of Knee Extension Strength and Visual Acuity on Gait}

In examining the knee extension strength and visual acuity factors, the superior KES group had significantly greater values in walking speed, cadence, and step length but smaller values in stance time, swing time, double support time, step width, and walking angle, as compared to the inferior group. These characteristics are similar to gait change with age. However, a combined effect of KES and VA factors was not found.

Furthermore, KES is an important factor that affects gait, and similar results were found in previous studies $[31,33]$. It was considered that visual acuity factors would not strongly affect healthy elderly women's gait, 
although the measurement condition must be considered.

In conclusion, the elderly with both a KES decrease and knee joint pain, as compared to the elderly with neither factor, have markedly different gait properties; e.g., decrease in walking speed, cadence, and step length, increase of stance time and walking angle. In addition, subjective visual acuity problems and knee joint pain have very little effect on gait during short, level walking. Knee extension strength affects gait and plays an important role in gait change.

\section{REFERENCES}

[1] Yamazaki, K., Kushida, K., Okui, K., Suzuki, M., Suzuki, K., Tamura, T., Saitoh, I. and Inoue, T. (1998) Investigation of factors to "bedridden" from "fractures". Osteoporosis Japan, 6, 265-268.

[2] Lord, S.R., Ward, J.A., Williams, P. and Anstey, K. (1994) Physiological factors associated with falls in older community-dwelling women. Journal of the American Geriatrics Society, 42, 1110-1117.

[3] Nevitt, M.C. (1997) Gait disorders of aging. LippincottRaven, Philadelphia, New York.

[4] Murray, M.P., Kory, R.C. and Clarkson, B.H. (1969) Walking patterns in healthy old men. Journal of Gerontology, 24, 169-178. doi:10.1093/geronj/24.2.169

[5] Murray, M.P., Kory, R.C. and Sepic, S.B. (1970) Walking patterns of normal women. Archives of Physical Medicine and Rehabilitation, 51, 637-650.

[6] Kaneko, M., Morimoto, Y., Kimura, M., Fuchimoto, K. and Fuchimoto, T. (1991) A kinematic analysis of walking and physical fitness in elderly women. Canadian Journal of Sport Sciences, 16, 223-228.

[7] Guralnik, J.M., Simonsick, E.M., Ferrucci, L., Glynn, R.J., Berkman, L.F., Blazer, D.G., Scherr, P.A. and Wallace, R.B. (1994) A short physical performance battery assessing lower extremity function: Association with selfreported disability and prediction of mortality and nursing home admission. Journal of Gerontology, 49, M85-M94. doi:10.1093/geronj/49.2.M85

[8] Guralnik, J.M., Ferrucci, L., Simonsick, E.M., Salive, M.E. and Wallage, R.B. (1995) Lower-extremity function in persons over the age of 70 years as a predictor of subsequent disability. New England Journal of Medicine, 332, 556-561. doi:10.1056/NEJM199503023320902

[9] Van Swearingen, J.M., Paschal, K.A., Bonino, P. and Chen, T.W. (1997) Assessing recurrent fall risk of communitydwelling, frail older veterans using specific tests of mobility and the physical performance test of function. Journals of Gerontology Series A, Biological Sciences and Medical Sciences, 53A, M457-M464. doi:10.1093/gerona/53A.6.M457

[10] Montoye, H.J. and Lamphiear, D.J. (1977) Grip and arm strength in males and females, age 10 to 69 . Research Quarterly, 48, 109-120.

[11] Frontera, W.R., Hughes, V.A., Lutz, K.J. and Evans, W.J. (1991) A cross-sectional study of muscle strength and mass in 45- to 78-yr-old men and women. Journal of Applied Physiology, 71, 644-650.

[12] Murray, M.P., Duthie Jr., E.H., Gambert, S.R., Sepic, S.B. and Mollinger, L.A. (1985) Age-related differences in knee muscle strength in normal women. Journal of Gerontology, 40, 275-280. doi:10.1093/geronj/40.3.275

[13] Singh, M.M. and Malhotra, H.S. (2003) Falls in the elderly-clinician's approach. Journal of the Indian Medical Association, 101, 420, 422, 424.

[14] Elliott, D.B., Bullimore, M.A., Patla, A.E. and Whitaker, D. (1996) Effect of a cataract simulation on clinical and real world vision. British Journal of Ophthalmology, 80, 799-804. doi:10.1136/bjo.80.9.799

[15] Moe-Nilssen, R., Helbostad, J.L., Akra, T., Birdedal, L. and Nygaard, H.A. (2006) Modulation of gait during visual adaptation to dark. Journal of Motor Behavior, 38 118-125. doi:10.3200/JMBR.38.2.118-125

[16] Klein, B.E.K., Moss, S.E., Klein, R., Lee, K.E. and Cruickshanks, K.J. (2003) Associations of visual function with physical outcomes and limitations 5 years later in an older population. Ophthalmology, 110, 644-650. doi:10.1016/S0161-6420(02)01935-8

[17] Nevitt, M., Commings, S., Kidd, S. and Black, D. (1989) Risk factors for recurrent non-syncopal falls. Journal of the American Medical Association, 261, 2663-2668. doi:10.1001/jama.1989.03420180087036

[18] Al-Zaharni, K.S. and Bakheit, A.M. (2002) A study of the gait characteristics of patients with chronic osteoarthritis of the knee. Disability and Rehabilitation, 24, 275-280. doi:10.1080/09638280110087098

[19] Bejek, Z., Paroczai, R., Illyes, A. and Kiss, R.M. (2006) The influence of walking speed on gait parameters in healthy people and in patients with osteoarthritis. Knee Surgery, Sports Traumatology, Arthroscopy, 14, 612-622. doi:10.1007/s00167-005-0005-6

[20] Kaufman, K.R., Hughes, C., Morrey, B.F., Morrey, M. and An, K.N. (2001) Gait characteristics of patients with knee osteoarthritis. Journal of Biomechanics, 34, 907-915. doi:10.1016/S0021-9290(01)00036-7

[21] McAlindon, T.E., Cooper, C., Kirwan, J.R. and Dieppe, P.A. (1992) Knee pain and disability in the community. Rheumatology, 31, 189-192. doi:10.1093/rheumatology/31.3.189

[22] Peat, G., McCarney, P. and Croft, P. (2001) Knee pain and osteoarthritis in older adults: A review of community burden and current use of primary health care. Annals of Rheumatic Diseases, 60, 91-97. doi:10.1136/ard.60.2.91

[23] Dieppe, P. and Lim, K. (1998) Osteoarthritis and related disorders: Clinical features and diagnostic problems. In: Dlippel, J.H. and Kieppe, P.A., Eds., Rheumatology, 2nd Edition, Mosby, London.

[24] Noro, M., Naito, K., Torii, S., Oka, K. and Nakamura, Y. (2007) Effects of knee pain improvement programs in middle- and older-aged women with knee pain. Japanese Journal of Physical Fitness and Sports, 56, 501-508.

[25] Demura, T. and Demura, S. (2010) Relationship among gait parameters while walking with varying loads. Journal of Physiological Anthropology, 29, 29-34. 
doi:10.2114/jpa2.29.29

[26] Nishijima, T., Koyama, R., Naito, I., Hatakeyama, S., Yamasaki, H. and Oku, T. (2004) Relationship between knee extension muscle strength and walking ability in the elderly patients. Rigakuryoho Kagaku, 19, 95-99. doi:10.1589/rika.19.95

[27] Demura, S., Sato, S., Yokoya, T. and Sato, T. (2010) Examination of useful items for the assessment of fall risk in the community-dwelling elderly Japanese population. Environmental Health and Preventive Medicine, 15, 169179. doi:10.1007/s12199-009-0124-7

[28] Blain, H., Vuillemin, A., Teissier, A., Hanesse, B., Guillemin, F. and Jeandel, C. (2001) Influence of muscle strength and body weight and composition on regional bone mineral density in healthy women aged 60 years and over. Gerontology, 47, 207-212. doi:10.1159/000052800

[29] Slemenda, C., Heilman, D.K., Brandt, K.D., Katz, B.P., Mazzuca, S.A., Braunstein, E.M. and Byrd, D. (1998) Reduced quadriceps strength relative to body weight. Arthritis and Rheumatism, 41, 1951-1959. doi:10.1002/1529-0131(199811)41:11<1951::AID-ART9 $>3.0 . \mathrm{CO} ; 2-9$
[30] Oliveria, S.A., Felson, D.T., Cirillo, P.A., Reed, J.I. and Walker, A.M. (1999) Body weight, body mass index, and incident symptomatic osteoarthritis of the hand, hip, and knee. Epidemiology, 10, 161-166. doi:10.1097/00001648-199903000-00013

[31] Yamagishi, T. and Tokuda, T. (1975) Geriatric gait-Photometric analysis. Japanese Journal of Rehabilitation Medicine, 12, 97-104. doi:10.2490/jijm1963.12.97

[32] Patla, A.E. (1997) Understanding the roles of vision in the control of human locomotion. Gait and Posture, 5, 54-69. doi:10.1016/S0966-6362(96)01109-5

[33] Hageman, P.A. and Blanke, D.J. (1986) Comparison of gait of young women and elderly women. Physical Therapy, 66, 1382-1387.

[34] Daubney, M.E. and Culham, E.G. (1999) Lower-extermity muscle force and balance performance in adults aged 65 years and older. Physical Therapy, 79, 1177-1185.

[35] Wolfson, L., Judge, J., Whipple, R. and King, M. (1995) Strength is a major factor in balance, gait, and the occurrence of falls. Journal of Gerontology, 50, 64-67. 